\title{
Hematological Side Effects of Immune Checkpoint Inhibitors: The Example of Immune-Related Thrombocytopenia
}

OPEN ACCESS

Edited by: Brian Gabrielli,

The University of Queensland,

Australia

Reviewed by:

Daniel Olive,

Aix-Marseille Université, France Jacques Barbet,

Arronax, France

*Correspondence:

Roser Calvo

roser.calvo@astrazeneca.com

Specialty section:

This article was submitted to Pharmacology of Anti-Cancer Drugs,

a section of the journal

Frontiers in Pharmacology

Received: 21 November 2018

Accepted: 10 April 2019

Published: 26 April 2019

Citation:

Calvo R (2019) Hematological Side Effects of Immune Checkpoint Inhibitors: The Example of Immune-Related

Thrombocytopenia.

Front. Pharmacol. 10:454. doi: 10.3389/fphar.2019.00454

\section{Roser Calvo* \\ Patient Safety, Safety Science, AstraZeneca Pharmaceuticals, Gaithersburg, MD, United States}

Immune-related hematological adverse events are amongst the rare but potentially life-threatening complications of immune checkpoint inhibitors. The spectrum of these toxicities is broadening as the number of patients exposed to these agents is increasing. Yet, they are still relatively unknown to many clinicians, possibly due to a lack of specific diagnostic criteria, which poses a challenge for their recognition and proper reporting, and partly due to their low incidence, often too low to be noted in most clinical trial publications. Since early detection and prompt intervention are crucial to prevent fatal consequences, it is of outmost importance that medical staff and patients be aware of these potential toxicities and learn to recognize and treat them adequately. This publication outlines strategies and offers guidance on the detection, diagnosis, risk assessment, monitoring and management of immune-related thrombocytopenia, a relatively common example of immune-related hematological toxicity of immune checkpoint inhibitors. Keywords: immune-related thrombocytopenia, immune-related adverse events, ir-AEs, immune checkpoint
inhibitors, immune-related hematological adverse events, immune thrombocytopenia

\section{INTRODUCTION}

Immune checkpoints inhibitors (ICIs) targeting PD-1/PD-L1 (programmed cell death receptor-1 or ligand-1) and CTLA-4 (lymphocyte-associated protein 4) have been associated with a growing list of autoimmune-like safety complications known as immune-related adverse events (ir-AEs), which can affect virtually any organ, mainly skin, gastrointestinal, hepatic, pulmonary, mucocutaneous, endocrine, and less frequently others including the hematological system.

With the increasing number of approved ICIs, new indications, and number of patients exposed to them, the repertoire of hematological ir-AEs (hem-irAEs) now extends to entities as varied as pure red cell aplasia (Gordon et al., 2009; Nair et al., 2016; Yuki et al., 2017), aplastic anemia/bone marrow failure (Comito et al., 2017; Michot et al., 2017; Helgadottir et al., 2017; Meyers et al., 2018), hemophilia A (Delyon et al., 2011; Lozier, 2012), acute thrombosis (Kunimasa et al., 2018), large granular lymphocytosis (Wei et al., 2012), hemophagocytic lymphohistiocytosis (Sadaat and Jang, 2018), macrophage activation syndrome (Malissen et al., 2017), eosinophilia (Bernard-Tessier et al., 2017), and hematological cytopenias affecting one or more hematological cell lines. Literature reports include cases of ir-neutropenia (Akhtari et al., 2009; Wei et al., 2012; Simeone et al., 2014; Wozniak et al., 2015; Sun et al., 2018), autoimmune hemolytic anemia (Kong et al., 2016; 
Nair et al., 2016; Palla et al., 2016; Schwab et al., 2016; Cooling et al., 2017; Khan et al., 2017; Tardy et al., 2017; Sun et al., 2018), ir-thrombocytopenia (ir-TCP) (Chung et al., 2010; Ahmad et al., 2012; Hilmi Atay et al., 2015; Kopecky et al., 2015; Solomon, 2015; Bagley et al., 2016; Inadomi et al., 2016; Kanameishi et al., 2016; Karakas et al., 2017; Le Burel et al., 2017; Pföhler et al., 2017; Shiuan et al., 2017; Jotatsu et al., 2018; Sun et al., 2018), and pancytopenia ( $\mathrm{Ku}$ et al., 2010; Di Giacomo et al., 2011; du Rusquec et al., 2014). Although hem-irAEs are rare, with ir-cytopenias reported with $\mathrm{PD}-1 / \mathrm{PD}-\mathrm{L} 1$ inhibitors at a frequency of $0.5 \%$ for CTCAE (Common Terminology Criteria for Adverse Events) grade $\geq 2$ events (Delanoy et al., 2019), they can be life-threatening and warrant early recognition and appropriate patient management to prevent potentially fatal outcomes. This review focuses specifically on ir-TCP as the most common type of hem-irAEs along with autoimmune hemolytic anemia and neutropenia, each occurring in $26 \%$ of patients with a reported hem-irAE during $\mathrm{PD}-1 / \mathrm{PD}-\mathrm{L} 1$ treatment registered in three French pharmacovigilance databases (Delanoy et al., 2019). Moreover, compared with TCP of conventional anticancer drugs, clinicians are less familiar with ir-TCP, which may lead to misdiagnosis of an entity that is clinically serious and for which delaying adequate care could lead to a worse prognosis. Despite the noted limitations due to the rarity of ir-TCP and consequently the retrospective nature of most series from which data for this publication is extracted, we hope this review will increase the physician's familiarity with clinical aspects of ir-TCP and algorithms for optimal management and minimization of this toxicity.

Mechanistically, ir-AEs are thought to be caused by a reinvigoration of exhausted T-cells once the ICI exerts the desired effect on the PD-1/PD-L1 or CTLA-4 pathway, evoking inflammation and ultimately leading to the occurrence of irAEs. Other immune cells may play a role, including B cells that produce antibodies that may mediate the toxicity. Although the precise pathogenesis of ir-TCP is unclear, the reaction is thought to be triggered by ICI-induced antiplatelet antibody production via an autoimmunity activation, which is supported by high levels of platelet-associated autoantibodies in many patients with ir-TCP (Pföhler et al., 2017; Jotatsu et al., 2018; Leroy et al., 2018).

While TCP of unspecified etiology is relatively frequent with ICIs (Ansell et al., 2015), ir-TCP is reported at an incidence of around 1-2\% (Chung et al., 2010; Friedman et al., 2016; Kourie et al., 2016; Shiuan et al., 2017; Le Burel et al., 2017; Sun et al., 2018). Because there may be alternative explanations for the TCP, there is a reasonable risk that ir-TCP may be under-recognized and treatment delayed, with an inherent risk of potentially fatal bleeding complications. There is increasing need for guidance to aid clinicians and investigators to anticipate, recognize, mitigate, monitor, and manage suspected cases of ir-TCP during ICI therapy. This paper offers a review of the current available reports in the literature (Chung et al., 2010; Ahmad et al., 2012; Hilmi Atay et al., 2015; Kopecky et al., 2015; Solomon, 2015; Bagley et al., 2016; Inadomi et al., 2016; Kanameishi et al., 2016; Karakas et al., 2017; Le Burel et al., 2017; Pföhler et al., 2017; Shiuan et al., 2017; Jotatsu et al., 2018).

\section{HOW SHOULD WE DEFINE AND DIAGNOSE IMMUNE-RELATED THROMBOCYTOPENIA?}

A practical definition of ir-TCP would enhance this AE recognition, optimal patient management, and case reporting. The challenge is that ir-TCP can mimic virtually any other type of TCP, with no specific biomarkers or diagnostic tests, making it a diagnosis of exclusion after ruling out other more common causes of TCP. A broad definition (e.g., "TCP following treatment with an ICI that is not attributable to another cause and/or has a clinical phenotype that suggests an immune event") may be helpful if supported by certain clinical factors that point to an underlying autoimmunity, such as those provided in Table 1. At a practical level, the possibility of an underling ir-TCP should always be considered with any new unexplained decrease in platelet counts, particularly when work-up does not uncover other common causes of TCP. Consistent with other irAEs, ir-TCP develops generally within the first 12 weeks of ICI initiation (Shiuan et al., 2017; Delanoy et al., 2019) but onset can occur at any time: at the beginning, a few months into treatment, and after immunotherapy termination. Delayed onset cases have been reported as late as two months after the cessation of therapy (Hilmi Atay et al., 2015; Leroy et al., 2018).

\section{PREDICTIVE BIOMARKERS OF IMMUNE-RELATED THROMBOCYTOPENIA}

No predictive biomarkers can reliably identify patients at risk of ir-TCP. Upregulation of PD-1 expression on B-cells prior to ICI treatment (Kanameishi et al., 2016), the detection of autoantibodies (e.g., antinuclear antibodies, lupus anticoagulant, anti-cardiolipin, antiphospholipid, rheumatoid factor and anti-cyclic citrullinated peptide) (Le Burel et al., 2017), and the presence of specific single nucleotide polymorphisms of PD-1 and CTLA4 possibly associated with certain autoimmune diseases including immune thrombocytopenic purpura (ITP) (e.g., PD-1 +7209 C/T SNP and an increased susceptibility to ITP, CTLA4 -1577 A/G and CT60 A/G associated with ITP severity, and PD-D1 -606 G/A and +63379 $\mathrm{C} / \mathrm{T}$ associated with the patient's ITP response to prednisolone) (Kasamatsu et al., 2018), have been postulated as potential biomarkers. However, these findings are not sufficiently conclusive to trigger any practical recommendation and it is unknown whether they can be extrapolated to the risk of ir-TCP.

\section{RISK FACTORS FOR IMMUNE-RELATED THROMBOCYTOPENIA}

Various risk factors may increase the susceptibility to develop ir-AEs with ICI therapy. Prior immune-related toxicity with 
a previous course of immunotherapy (Champiat et al., 2016) and a personal or family history of autoimmune disorders (Bagley et al., 2016; Johnson et al., 2016; Gutzmer et al., 2017; Le Burel et al., 2017; Menzies et al., 2017; Pföhler et al., 2017; Jotatsu et al., 2018) are currently being assessed; if present, the patient's benefit-risk should be reassessed prior to use of ICIs. Immune-related cytopenias that develop during ICI treatment are common in patients with a prior diagnosis of psoriasis, vitiligo, ITP, or Hashimoto's thyroiditis, the presence of cold agglutinins, or prior treatment with temozolomide chemotherapy (Ku et al., 2010; Ahmad et al., 2012; du Rusquec et al., 2014), perhaps supporting the link with autoimmune diseases.

Certain concomitant medications that affect the immune system (e.g., steroids, allopurinol, non-steroidal antiinflammatory drugs, salicylates, metformin, etc.) or that are associated with drug-induced immune TCP (e.g., quinine, quinidine, cotrimoxazole, gold salts, oral antidiabetics, colchicine, etc.) may increase the potential for drug-associated autoimmunity (Solomon, 2015; Champiat et al., 2016). However, these risk factors are not determinant and, thus far, risk mitigation strategies cannot be based solely on excluding them.

TABLE 1 | Diagnostic criteria suggestive of immune-related thrombocytopenia.

\section{(1) Absence of any other factors likely to cause TCP}

\section{Non-immune}

Prior or concomitant chemo-radiotherapy. Bone marrow tumor infiltration. Hematological malignancies (MDS, MPD).

Platelet sequestration (hypersplenism, liver diseases)

Platelet consumption (TMA, TTP, DIC)

\section{(2) Severe TCP (platelet nadir $<20,000 / \mathrm{mm}^{3}$ )}

\section{Concomitant chemotherapy may cause similar TCP severity}

\section{(3) Quick rate of decline of platelet counts}

Decrease of platelet counts over a few days or weeks with no alternative explanation

\section{(4) Positive detection of antiplatelet antibodies}

Most drug-induced immune TCPs are caused by drug-dependent antibodies specific for the drug structure that react to platelets only in the presence of the drug. Antiplatelet antibody testing may help to document the cause of TCP, the causative drug, and which platelet glycoprotein is being recognized [allb/B3 integrin (GPIlb/Illa), GPIb/IX, etc.,] but is technically demanding, not useful in the immediate clinical decision making, and there may be false negatives even when clinical scenario is highly suggestive of drug-induced immune TCP

\section{(5) Bone marrow aspirate and/or biopsy compatible with peripheral (not central) TCP}

Increased number of megakaryocytes without abnormal cells in bone marrow, with increased percentage of immature platelets in peripheral blood, indicating increased thrombopoiesis

(6) Temporal relationship with onset of TCP after ICI administration

Most reports describe an early onset within days or weeks of initial cycles although later onset (even months after treatment cessation) has been described. It may occur after initial ICl exposure or after re-exposure following a treatment interruption

\section{(7) Recovery of platelets after stopping the ICI}

Complete and sustained recovery of platelet counts spontaneously after permanent interruption of the ICl, with or without additional steroids or immunosuppressants (8) Effectiveness of corticosteroids and immunosuppressants

Quick and effective response to steroids, intravenous immunoglobulins or other immunosuppressors such as cyclosporine or rituximab

\section{(9) Ineffective platelet transfusions}

Failure of platelet transfusions to produce a sustained increase in platelet count because the antiplatelet antibodies cause destruction of the transfused platelets

\section{(10) Personal or family history of autoimmune disease}

Psoriasis, vitiligo, ITP, and Hashimoto thyroiditis have been described in ICl-treated patients diagnosed with ir-TCP

\section{(11) Positive autoantibodies in the immunologic screening}

Antinuclear antibodies, lupus anticoagulant, anti-cardiolipin/antiphospholipid autoantibodies, and others have been detected in the serum of ICI-treated patients who developed ir-TCP. However, positive results are not confirmatory of autoimmune disease (ir-TCP) because autoantibodies may be present in healthy individuals and in patients with non-autoimmune diseases, but provide further evidence

\section{(12) Positive direct antiglobulin test (Direct Coombs Test)}

May indicate simultaneous autoimmune hemolytic anemia (Evans Syndrome) and be supportive of a diagnosis of TCP of immune origin

\section{(13) Occurrence of other ir-AEs in the same patient}

Prior or concomitant neurological, endocrine, gastrointestinal, liver and skin ir-AEs have been described in ICI-treated patients diagnosed with ir-TCP and may raise clinical suspicion of a diagnosis of ir-TCP

TCP, thrombocytopenia; ir-TCP, immune-related thrombocytopenia; MDS, myelodysplastic syndrome; MPD, myeloproliferative diseases; TMA, thrombotic microangiopathy; TTP, thrombotic thrombocytopenic purpura; DIC, disseminated intravascular coagulation; HIV, human immunodeficiency virus; HCV, hepatitis C virus;

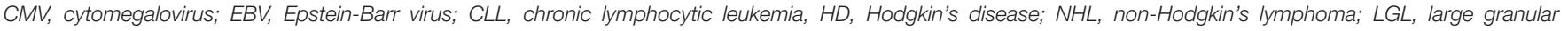

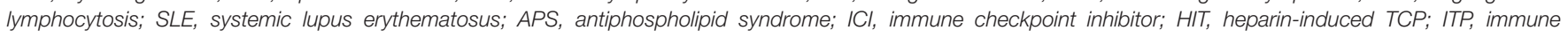
thrombocytopenia; IVIG, intravenous immunoglobulin; AEs, adverse events; PD1/PD-L1, programmed cell death receptor-1 or ligand-1. 


\section{STANDARD ASSESSMENTS TO EVALUATE SUSPECTED IMMUNE-RELATED THROMBOCYTOPENIA}

The initial standard work-up when ir-TCP is suspected should include a patient's detailed medical and family history to help inform on potential risk factors such as history of autoimmunity or certain prior or concomitant medications, a nutritional evaluation and physical examination, and a basic laboratory panel with complete blood count $(\mathrm{CBC})$ and differential, peripheral blood smear, reticulocyte count, direct antiglobulin test, and infection panel. When results are still inconclusive, a bone marrow examination may be helpful to differentiate central versus peripheral TCP. These first-tier evaluations are usually sufficient to establish the most likely diagnosis and guide clinical management decisions. Second-tier assessments, based on initial lab results and clinical suspicion, can provide further evidence to exclude or confirm alternative TCP causes such as heparininduced TCP (anti-PF4 antibodies), certain infections (additional virus/bacterial panel), disseminated intravascular coagulation (coagulation parameters), thyroid dysfunction (antithyroid antibodies with thyroid function), and importantly, immune TCP induced by concomitant medications (specific drug-dependent antiplatelet antibodies). Table 2 summarizes the recommended evaluations. While standard assessments should be performed as soon as an unexplained drop in platelet count is detected, the timing for complementary assessments is usually guided by the initial lab results and the patient's clinical evolution.

\section{SIGNAL DETECTION AND ASSESSMENT OF IMMUNE-RELATED THROMBOCYTOPENIA IN CLINICAL DEVELOPMENT PROGRAMS}

\section{Signal Detection}

Safety assessment is an integral part of any clinical development program. To identify a safety signal of ir-TCP in ICI clinical programs, it is necessary to first define the platelet threshold that constitutes TCP.

First, 100,000 cells $/ \mathrm{mm}^{3}$ is the cut-off to define primary immune TCP (Rodeghiero et al., 2009), which could certainly be used for ir-TCP signal detection. This threshold avoids

TABLE 2 | Recommended investigations for suspected immune-related thrombocytopenia.

Clinical evaluation

\section{Parameter}

Patient history

Family history

Nutritional evaluation

Physical examination

\author{
Points to consider \\ Personal history of autoimmune disease (Hashimoto's thyroiditis, ITP, vitiligo, psoriasis); prior \\ chemo-radiotherapy or lymphocyte-depleting therapy (fludarabine, anti-thymocyte globulin, \\ corticosteroids) \\ History of autoimmune disease \\ Iron, folate, vitamin B12 deficiency \\ Splenomegaly, hepatomegaly, purpura, petechiae
}

Standard investigations

Investigation

Complete blood count

Peripheral blood smear

Reticulocyte count

Direct antiglobulin test [direct Coombs test]

Infection panel

Bone marrow examination (aspirate/biopsy)

\begin{abstract}
Points to consider
Other hematological cell lines decreased (coexistence of neutropenia and/or anemia)

Platelet clumping (pseudo-TCP), morphology of platelets (myelodysplastic syndrome)

Coexistence of autoimmune hemolytic anemia (Evans syndrome)

Coexistence of autoimmune hemolytic anemia (Evans Syndrome)

HIV, HCV, H. Pylori
\end{abstract}

Not always necessary; indicated when other investigations are not conclusive or if other cell lines are affected and there is concern for drug-induced aplastic anemia or bone marrow pathology

\section{Optional investigations}

\section{Investigation}

Platelet antibody identification panel/drug-dependent antiplatelet antibodies

Anti PF4

Additional virus panel

Coagulation parameters

Autoantibody screening

Antithyroid antibodies and thyroid function

\section{Points to consider}

Human platelet antibodies HPA-1a/b, HPA-2a/b, HPA-3a/b, HPA-4a, HPA-5a/b, GPIllb/lla, GPla/lla, GP Ib/IX, GPIV, and class I HLA

Heparin-induced TCP

Parvovirus B19, EBV, CMV

PT, aPTT, fibrinogen, D-dimers: rule out TMA/TP/DIC

Antinuclear antibodies and antiphospholipid autoantibodies (including lupus anticoagulant and anti-cardiolipin)

Coexisting autoimmune thyroid disorders (e.g., subclinical hypothyroidism associated with ITP: treating the underlying thyroid disorder may significantly improve platelet count)

HIV, human immunodeficiency virus; HCV, hepatitis C virus; TCP, thrombocytopenia; HPA, Human platelet antibodies; GP, glycoprotein; HLA, human leukocyte antigen; TMA, thrombotic microangiopathy; TTP, thrombotic thrombocytopenic purpura; DIC, disseminated intravascular coagulation; HIV, human immunodeficiency virus; HCV, hepatitis C virus; HBV, hepatitis B virus; CMV, cytomegalovirus; EBV, Epstein-barr virus; HIT, heparin-induced TCP; ITP, immune thrombocytopenia. 
concerns over cell count reductions where platelets remain above 100,000 cells $/ \mathrm{mm}^{3}$ which usually do not carry any risk for serious bleeding. In line with this platelet cut-off value, the American Society of Clinical Oncology (ASCO) guidelines for the management of ir-AEs in patients treated with ICIs provide specific recommendations for ir-TCP when platelet counts decrease below 100,000 cells $/ \mathrm{mm}^{3}$. Hence, a reduction in absolute platelet counts below 100,000 cells $/ \mathrm{mm}^{3}$ seems a reasonable threshold to use for thrombocytopenia signal detection.

Secondly, in the context of immunotherapy, certain platelet count decreases relative to baseline values should also constitute a warning for the physician, even if the platelet count remains within the normal range. This may be particularly useful for signal detection in patients who initiate treatment with a certain degree of TCP as a sequela from prior treatments, for whom a new onset ir-TCP may be partially masked by the pre-existing TCP. In this context, a relative decrease more than $50 \%$ over the platelet baseline value may be a useful indicator of a potential newly developing ir-TCP.

Therefore, for optimal TCP signal detection and platelet monitoring during ICI therapy, both an unexplained decrease in absolute platelet counts below 100,000 cells $/ \mathrm{mm}^{3}$ and/or a reduction of $\geq 50 \%$ from the individual patient's baseline level are important, and either one should warrant a closer evaluation and more frequent patient monitoring.

\section{Search Strategy for Safety Data Retrieval in Clinical Development Programs}

For investigation of drug safety concerns and data retrieval and presentation from individual clinical trials and across clinical development programs, it is necessary to identify and aggregate data from all ir-TCP reported cases. A comprehensive search of the safety databases should include a combination of Preferred Terms (PTs) and Standardized MedDRA Queries (SMQs) from the Medical Dictionary for Regulatory Activities (MedDRA). For example, based on the MedDRA browser version at the time of writing this article, the search should include the "haematopoietic cytopenias affecting more than one type of blood cell" (SMQ), the "haematopoietic thrombocytopenia" (SMQ), and two additional PTs: "immune thrombocytopenic purpura" and "thrombocytopenic purpura." Of note, the search should be revised as new MedDRA versions become available since new terms may be added and others become obsolete.

\section{Signal Assessment Causality Assessment}

Causal attribution of TCP to the ICI during therapy may be confounded by the underlying malignancy (disease progression and bone marrow infiltration) and concomitant medications (drug-related TCP), among others. Table 1 proposes a criteria checklist suggestive of ir-TCP during ICI therapy. Any patient who develops unexplained TCP should be evaluated for these factors and a possible diagnosis of ir-TCP ruled out. When the diagnosis of drug-induced ir-TCP is highly suspected in a pluri-medicated patient, it may be virtually impossible to be identify the causative drug. Investigation of drug-dependent antiplatelets antibodies in serum and platelet-bound antibodies at the time of diagnosis of TCP may help confirm the etiology but requires significant time and is not useful for management decisions.

\section{Systematic Evaluation Across Cases: Adverse Event Reporting Versus Laboratory Values}

TCP is relatively frequent in oncology clinical trials of ICIs, but it may not always be accurately or consistently reported as an $\mathrm{AE}$ when applicable. For this reason, evaluation of changes in laboratory parameters during treatment (e.g., study and program-level shift tables for platelet counts from baseline to the worst and to the last platelet value) may provide more reliable and complementary information on the compound's potential to cause TCP and its reversibility.

\section{Severity Grading of Immune-Related Thrombocytopenia}

The CTCAE grading is helpful in guiding dosing decisions in oncology practice and in standardizing the reporting of toxicity across clinical development programs. However, its reliability for evaluating the occurrence and severity of ir-TCP during ICI therapy is questionable as it is based on absolute platelet counts and does not reflect relative changes in platelet counts from baseline. Despite this limitation, there is currently no alternative tool for measuring platelet shifts possibly associated to ICIs.

\section{RISK MANAGEMENT AND POTENTIAL MITIGATION STRATEGIES}

\section{Screening for Patients "at Risk" and Risk Mitigation}

The lack of laboratory or clinical parameters that can predict the risk of developing ir-TCP or its severity prevents implementing reliable de-risking strategies. A certain predisposition to hemirAEs during PD1/PD-L1 therapy in patients with a personal or family history of autoimmune disease raises the possibility of screening for at-risk patients by testing for certain autoantibodies (Le Burel et al., 2017). An additional de-risking strategy could consist of intensifying the clinical (more frequent patient visits) and biological (more frequent $\mathrm{CBCs}$ ) monitoring in patients who present:

(a) A positive autoantibodies screening prior to ICI treatment (e.g., antinuclear antibodies, lupus anticoagulant, anticardiolipin, antiphospholipid autoantibodies, rheumatoid factor, and anti-cyclic citrullinated peptide).

(b) An increase in absolute eosinophil and lymphocyte count at 1 month after ICI therapy initiation, which have been correlated to an increased risk of all ir-AEs (Schindler et al., 2014; Diehl et al., 2017), possibly based on the immunological functional links between IL-17 and eosinophils, and their correlation with ir-AEs.

However, there is not enough evidence to support a robust association between these biomarkers and risk of ir-TCP, or to reliably use them for patient selection and risk minimization. 
Therefore, patients should not be denied treatment with ICIs based on positive results because in cancer patients the risk/benefit balance should go toward the cancer treatment.

Finally, a key component of risk mitigation is to ensure that both physician and patient are aware of the possible occurrence of less common yet potentially life-threatening ir-AEs such as ir-TCP. Patients should be educated to recognize clinical signs and symptoms of a possible developing TCP such as mucosal bleeding, petechiae, macroscopic hematuria, buccal hemorrhagic bulla, purpura or any type of abnormal bleeding, and should be informed that most of these events are reversible if detected early and properly treated.

\section{Patient Monitoring}

Patients should be under close monitoring at the beginning, during, and even after ICI treatment discontinuation as ir-TCP may occur at any time and there are no predictive biomarkers of risk. Some experts recommend continued monitoring until 1 year after ICI discontinuation (Champiat et al., 2016).

Clinicians should monitor for signs/symptoms of TCP (easy bruising, petechiae, spontaneous mucocutaneous bleeding) at each visit and advise patients to immediately report abnormal bleeding. A CBC should be performed at baseline and prior to each dose administration, and counts should always be compared with baseline values to detect any clinically relevant shift in platelet counts. A more frequent $\mathrm{CBC}$ may be considered when (a) platelets drop below $100,000 / \mathrm{mm}^{3}$ or there is a reduction of more than $50 \%$ from baseline values (e.g., depending on workup results, consider monitoring once weekly); and (b) the rate of platelet decline is very rapid and there is clinical suspicion that a severe ir-TCP may be developing (e.g., monitor twice weekly or more often if clinically indicated).

Once the possible diagnosis of ir-TCP is established, the patient should be monitored for signs of bleeding (e.g., mucosal bleeding, petechiae, hematuria, buccal hemorrhagic bulla, and very specially "wet purpura" which is associated with a high risk of severe bleeding). CBCs should be performed as clinically indicated until resolution of the TCP or until reaching a stable platelet count, preferably $\geq 100,000 / \mathrm{mm}^{3}$ or baseline values in those patients who had some degree of TCP before treatment initiation.

In terms of event resolution, clinicians should be aware that the strategy and time needed for ir-TCP recovery can highly vary across patients, with platelet counts rising in some patients as early as day 2 with normalization by day 4 (Le Roy et al., 2016), and others necessitating up to 3 months for normalization (Pföhler et al., 2017). Although mild ir-TCP may resolve spontaneously with temporary or permanent treatment discontinuation (Shiuan et al., 2017; Jotatsu et al., 2018), the most comprehensive series characterizing hem-irAEs associated with anti PD-1/PD-L1therapy recently published by Delanoy et al. (2019) described that $78 \%$ of ir-TCP cases were grade $4,100 \%$ of patients received steroids, $67 \%$ received additional intravenous immunoglobulin (IVIG), and $22 \%$ did not respond to steroids or IVIG and required treatment with thrombopoetin agonists or rituximab.
Additionally, this study provided preliminary data about the safety of ICI rechallenge in patients with ir-TCP: $67 \%$ patients discontinued the ICI permanently and $33 \%$ of patients were rechallenged, of which $33 \%$ had a recurrence of ir-TCP after the rechallenge. Although the data is too scarce to draw any definite conclusion, the risk of ir-TCP recurrence after rechallenge must be considered, and increased vigilance is warranted. In this series, $78 \%$ of patients had resolution of ir-TCP at final follow-up.

\section{TOXICITY MANAGEMENT GUIDELINES OF IMMUNE-RELATED THROMBOCYTOPENIA}

Various guidelines are available for the management of irAEs including the European Society for Medical Oncology (Haanen et al., 2017), the Society for Immunotherapy of Cancer (Puzanov et al., 2017), and the ASCO (Brahmer et al., 2018) guidelines. On the basis of the ir-TCP severity, the ASCO guidelines recommend early suspension of the ICI when platelets reach $<75,000 / \mathrm{mm}^{3}$, with initiation of steroids if a quicker platelet count increase is warranted (e.g., abnormal bleeding). Of note, these recommendations are meant for ICIs administered alone and do not provide guidance for ICI combinations with agents with thrombocytopenic liability.

According to the ASCO guidelines, ir-TCP grade 1 $\left(<100,000 / \mathrm{mm}^{3}\right)$ should be managed by ICI continuation with close clinical follow-up and laboratory evaluation. Grade $2\left(<75,000 / \mathrm{mm}^{3}\right)$ requires ICI withholding but monitoring for improvement with interruption until resolution to grade 1, along with administration of oral prednisone with 24 weeks tapering and optional IVIG in conjunction with corticosteroids if a more rapid platelet increase is required. For grade $3\left(<50,000 / \mathrm{mm}^{3}\right)$ and grade $4\left(<25,000 / \mathrm{mm}^{3}\right)$, a hematology consult is indicated; treatment should be withheld until resolution to grade 1, with administration of high-dose corticosteroids (prednisone 1 to $2 \mathrm{mg} / \mathrm{kg} / \mathrm{d}$ or methylprednisolone 1 to $2 \mathrm{mg} / \mathrm{kg} / \mathrm{d}$ ) and optional IVIG if a more-rapid platelet increase is required, with permanent discontinuation of ICI there is no improvement. If previous corticosteroids and/or IVIG are unsuccessful, subsequent treatment may include rituximab, splenectomy, thrombopoietin receptor agonists, or more potent immunosuppression.

\section{CONCLUSION}

Hem-irAEs are potentially life-threatening complications of ICIs that require a high level of clinical suspicion. Ir-TCP is amongst the most frequently reported hem-irAEs. Due to its low incidence and the lack of specific diagnostic criteria, ir-TCP may be underdiagnosed and possibly underreported. The diagnosis is based on exclusion of other causes of TCP together with clinical features that suggest an immune event such as the refractoriness 
to platelet transfusions and response to corticosteroids. During clinical development, a systematic analysis of ir-TCP cases at clinical study and program level is of outmost importance to better characterize the TCP, understand the risk factors, support causality assessment, and optimize the risk management. In clinical practice, it is critical that both patients and medical personnel involved in ICI therapy be knowledgeable of this rare but potentially serious complication since prompt recognition and initiation of appropriate treatment is key to avoid severe bleeding complications.

\section{REFERENCES}

Ahmad, S., Lewis, M., Corrie, P., and Iddawela, M. (2012). Ipilimumab-induced thrombocytopenia in a patient with metastatic melanoma. J. Oncol. Pharm. Pract. 18, 287-292. doi: 10.1177/1078155211411001

Akhtari, M., Waller, E. K., Jaye, D. L., Lawson, D. H., Ibrahim, R., Papadopoulos, N. E., et al. (2009). Neutropenia in a patient treated with ipilimumab (anti-CTLA-4 antibody). J. Immunother. 32, 322-324. doi: 10.1097/CJI. 0b013e31819aa40b

Ansell, S. M., Lesokhin, A. M., Borrello, I., Halwani, A., Scott, E. C., Gutierrez, M., et al. (2015). PD-1 blockade with nivolumab in relapsed or refractory hodgkin's lymphoma. N. Engl. J. Med. 372, 311-319. doi: 10.1056/NEJMoa1411087

Bagley, S. J., Kosteva, J. A., Evans, T. L., and Langer, C. J. (2016). Immune thrombocytopenia exacerbated by nivolumab in a patient with non-small-cell lung cancer. Cancer Treat. Commun. 6, 20-23. doi: 10.1016/j.ctrc.2016.02.009

Bernard-Tessier, A., Jeanville, P., Champiat, S., Lazarovici, J., Voisin, A. L., Mateus, C., et al. (2017). Immune-related eosinophilia induced by anti-programmed death 1 or death-ligand 1 antibodies. Eur. J. Cancer 81, 135-137. doi: 10.1016/ j.ejca.2017.05.017

Brahmer, J. R., Lacchetti, C., Schneider, B. J., Atkins, M. B., Brassil, K. J., Caterino, J. M., et al. (2018). Management of immune-related adverse events in patients treated with immune checkpoint inhibitor therapy: american society of clinical oncology clinical practice guideline. J. Clin. Oncol. 36, 1714-1768.

Champiat, S., Lambotte, O., Barreau, E., Belkhir, R., Berdelou, A., Carbonnel, F., et al. (2016). Management of immune checkpoint blockade dysimmune toxicities: a collaborative position paper. Ann. Oncol. 27, 559-574. doi: 10.1093/ annonc/mdv623

Chung, K. Y., Gore, I., Fong, L., Venook, A., Beck, S. B., Dorazio, P., et al. (2010). Phase II study of the anti-cytotoxic T-lymphocyte-associated antigen 4 monoclonal antibody, tremelimumab, in patients with refractory metastatic colorectal cancer. J. Clin. Oncol. 28, 3485-3490. doi: 10.1200/JCO.2010.28. 3994

Comito, R. R., Badu, L. A., and Forcello, N. (2017). Nivolumab-induced aplastic anemia: a case report and literature review. J. Oncol. Pharm. Pract. 25, 221-225. doi: $10.1177 / 1078155217726159$

Cooling, L. L., Sherbeck, J., Mowers, J. C., and Hugan, S. L. (2017). Development of red blood cell autoantibodies following treatment with checkpoint inhibitors: a new class of anti-neoplastic, immunotherapeutic agents associated with immune dysregulation. Immunohematology 33, 15-21.

Delanoy, N., Michot, J. M., Comont, T., Kramkimel, N., Lazarovici, J., Dupont, R., et al. (2019). Haematological immune-related adverse events induced by antiPD-1 or anti-PD-L1 immunotherapy: a descriptive observational study. Lancet Haematol. 6, e48-e57. doi: 10.1016/S2352-3026(18)30175-3

Delyon, J., Mateus, C., and Lambert, T. (2011). Hemophilia a induced by ipilimumab. N. Engl. J. Med. 365, 1747-1748. doi: 10.1056/nejmc111 0923

Di Giacomo, A. M., Danielli, R., Calabro, L., Bertocci, E., Nannicini, C., Giannarelli, D., et al. (2011). Ipilimumab experience in heavily pretreated patients with melanoma in an expanded access program at the university hospital of siena (Italy). Cancer Immunol. Immunother. 60, 467-477. doi: 10.1007/s00262-0100958-2

Diehl, A., Yarchoan, M., Yang, T., Scott, B., Johnson, B. A., Hopkins, A., et al. (2017). Relationship of lymphocyte and eosinophil counts and immune-related adverse events in recipients of programmed death-1 (PD-1) inhibitor therapy: a

\section{AUTHOR CONTRIBUTIONS}

$\mathrm{RC}$ conceived the original idea, reviewed the literature, and wrote and edited the manuscript.

\section{ACKNOWLEDGMENTS}

The author thanks Stefan Sultana for his valuable editing and proofreading contributions.

single-center retrospective analysis. J. Clin. Oncol. 35:e14586. doi: 10.1200/jco. 2017.35.15_suppl.e14586

du Rusquec, P., Saint-Jean, M., Brocard, A., Peuvrel, L., Khammari, A., Quereux, G., et al. (2014). Ipilimumab-induced autoimmune pancytopenia in a case of metastatic melanoma. J. Immunother. 37, 348-350. doi: 10.1097/CJI. 0000000000000041

Friedman, C. F., Proverbs-Singh, T. A., and Postow, M. A. (2016). Treatment of the immune-related adverse effects of immune checkpoint inhibitors: a review. JAMA Oncol. 2, 1346-1353. doi: 10.1001/jamaoncol.2016.1051

Gordon, I. O., Wade, T., Chin, K., Dickstein, J., and Gajewski, T. F. (2009). Immune-mediated red cell aplasia after anti-CTLA-4 immunotherapy for metastatic melanoma. Cancer Immunol. Immunother. 58, 1351-1353. doi: 10. 1007/s00262-008-0627-x

Gutzmer, R., Koop, A., Meier, F., Hassel, J. C., Terheyden, P., Zimmer, L., et al. (2017). Programmed cell death protein-1 (PD-1) inhibitor therapy in patients with advanced melanoma and preexisting autoimmunity or ipilimumabtriggered autoimmunity. Eur. J. Cancer 75, 24-32. doi: 10.1016/j.ejca.2016. 12.038

Haanen, J. B. A. G., Carbonnel, F., Robert, C., Kerr, K. M., Peters, S., Larkin, J., et al. (2017). Management of toxicities from immunotherapy: ESMO clinical practice guidelines for diagnosis, treatment and follow-up†. Ann. Oncol. 28, iv119-iv142. doi: 10.1093/annonc/mdx225

Helgadottir, H., Kis, L., Ljungman, P., Larkin, J., Kefford, R., Ascierto, P. A., et al. (2017). Lethal aplastic anemia caused by dual immune checkpoint blockade in metastatic melanoma. Ann. Oncol. 28, 1672-1673. doi: 10.1093/annonc/ $\operatorname{mdx} 177$

Hilmi Atay, M., Kelkitli, E., and Yilmaz, B. (2015). Delayed severe thrombocytopenia due to Ipilimumab. J. BUON 20, 1641-1642.

Inadomi, K., Kumagai, H., Arita, S., Tsuruta, N., Takayoshi, K., Mishima, K., et al. (2016). Bi-cytopenia possibly induced by anti-PD-1 antibody for primary malignant melanoma of the esophagus: a case report. Medicine 95:e4283. doi: 10.1097/MD.0000000000004283

Johnson, D. B., Sullivan, R. J., Ott, P. A., Carlino, M. S., Khushalani, N. I., Ye, F., et al. (2016). Ipilimumab therapy in patients with advanced melanoma and preexisting autoimmune disorders. JAMA Oncol. 2, 234-240. doi: 10.1001/ jamaoncol.2015.4368

Jotatsu, T., Oda, K., Yamaguchi, Y., Noguchi, S., Kawanami, T., Kido, T., et al. (2018). Immune-mediated thrombocytopenia and hypothyroidism in a lung cancer patient treated with nivolumab. Immunotherapy 10, 85-91. doi: 10.2217/ imt-2017-0100

Kanameishi, S., Otsuka, A., Nonomura, Y., Fujisawa, A., Endo, Y., and Kabashima, K. (2016). Idiopathic thrombocytopenic purpura induced by nivolumab in a metastatic melanoma patient with elevated PD-1 expression on B cells. Ann. Oncol. 27, 546-547. doi: 10.1093/annonc/mdv580

Karakas, Y., Yuce, D., and Kilickap, S. (2017). Immune thrombocytopenia Induced by nivolumab in a metastatic non-small cell lung cancer patient. Oncol. Res. Treat. 40, 621-622. doi: 10.1159/000477968

Kasamatsu, T., Ino, R., Takahashi, N., Gotoh, N., Minato, Y., Takizawa, M., et al. (2018). PDCD1 and CTLA4 polymorphisms affect the susceptibility to, and clinical features of, chronic immune thrombocytopenia. Br. J. Haematol. 180, 705-714. doi: 10.1111/bjh.15085

Khan, U., Ali, F., Khurram, M. S., Zaka, A., and Hadid, T. (2017). Immunotherapyassociated autoimmune hemolytic anemia. J. Immunother. Cancer 5:15. doi: 10.1186/s40425-017-0214-9 
Kong, B. Y., Micklethwaite, K. P., Swaminathan, S., Kefford, R. F., and Carlino, M. S. (2016). Autoimmune hemolytic anemia induced by anti-PD-1 therapy in metastatic melanoma. Melanoma Res. 26, 202-204. doi: 10.1097/CMR. 0000000000000232

Kopecky, J., Trojanova, P., Kubecek, O., and Kopecky, O. (2015). Treatment possibilities of ipilimumab-induced thrombocytopenia-case study and literature review. Jpn. J. Clin. Oncol. 45, 381-384. doi: 10.1093/jjco/hyu222

Kourie, H. R., Awada, G., and Awada, A. H. (2016). Rare side-effects of checkpoint inhibitors. Curr. Opin. Oncol. 28, 295-305. doi: 10.1097/CCO. 0000000000000294

Ku, G. Y., Yuan, J., Page, D. B., Schroeder, S. E., Panageas, K. S., Carvajal, R. D., et al. (2010). Single-institution experience with ipilimumab in advanced melanoma patients in the compassionate use setting: lymphocyte count after 2 doses correlates with survival. Cancer 116, 1767-1775. doi: 10.1002/cncr.24951

Kunimasa, K., Nishino, K., Kimura, M., Inoue, T., Tamiya, M., Kumagai, T., et al. (2018). Pembrolizumab-induced acute thrombosis: a case report. Medicine 97:e10772. doi: 10.1097/MD.0000000000010772

Le Burel, S., Champiat, S., Mateus, C., Marabelle, A., Michot, J. M., Robert, C., et al. (2017). Prevalence of immune-related systemic adverse events in patients treated with anti-programmed cell death 1/anti-programmed cell death-ligand 1 agents: a single-centre pharmacovigilance database analysis. Eur. J. Cancer 82, 34-44. doi: 10.1016/j.ejca.2017.05.032

Le Roy, A., Kempf, E., Ackermann, F., Routier, E., Robert, C., Turpin, A., et al. (2016). Two cases of immune thrombocytopenia associated with pembrolizumab. Eur. J. Cancer 54, 172-174. doi: 10.1016/j.ejca.2015.10.073

Leroy, L., Lafarge, X., Blouin, L., Bijou, F., Durrieu, F., Olivier, E., et al. (2018). A fatal allo- and immune-mediated thrombocytopenia with a PD-L1 inhibitor. Ann. Oncol. 29, 514-515. doi: 10.1093/annonc/mdx693

Lozier, J. (2012). More on hemophilia a induced by ipilimumab. N. Engl. J. Med. 366, 280-281; author reply281.

Malissen, N., Lacotte, J., Du-Thanh, A., Gaudy-Marqueste, C., Guillot, B., and Grob, J. J. (2017). Macrophage activation syndrome: a new complication of checkpoint inhibitors. Eur. J. Cancer 77, 88-89. doi: 10.1016/j.ejca.2017.02.016

Menzies, A. M., Johnson, D. B., Ramanujam, S., Atkinson, V. G., Wong, A. N. M., Park, J. J., et al. (2017). Anti-PD-1 therapy in patients with advanced melanoma and preexisting autoimmune disorders or major toxicity with ipilimumab. Ann. Oncol. 28, 368-376. doi: 10.1093/annonc/mdw443

Meyers, D. E., Hill, W. F., Suo, A., Jimenez-Zepeda, V., Cheng, T., and Nixon, N. A. (2018). Aplastic anemia secondary to nivolumab and ipilimumab in a patient with metastatic melanoma: a case report. Exp. Hematol. Oncol. 7:6. doi: 10.1186/s40164-018-0098-5

Michot, J. M., Vargaftig, J., Leduc, C., Quere, G., Burroni, B., Lazarovici, J., et al. (2017). Immune-related bone marrow failure following anti-PD1 therapy. Eur. J. Cancer 80, 1-4. doi: 10.1016/j.ejca.2017.04.004

Nair, R., Gheith, S., and Nair, S. G. (2016). Immunotherapy-associated hemolytic anemia with pure red-cell aplasia. N. Engl. J. Med. 374, 1096-1097. doi: 10. 1056/nejmc1509362

Palla, A. R., Kennedy, D., Mosharraf, H., and Doll, D. (2016). Autoimmune hemolytic anemia as a complication of nivolumab therapy. Case Rep. Oncol. 9, 691-697. doi: 10.1002/hon.2338

Pföhler, C., Eichler, H., Burgard, B., Krecké, N., Müller, C. S. L., and Vogt, T. (2017). A case of immune thrombocytopenia as a rare side effect of an immunotherapy with pd1-blocking agents for metastatic melanoma. Transfus. Med. Hemother. 44, 426-428. doi: 10.1159/000479237

Puzanov, I., Diab, A., Abdallah, K., Bingham, C. O., Brogdon, C., Dadu, R., et al. (2017). Managing toxicities associated with immune checkpoint inhibitors: consensus recommendations from the society for immunotherapy of cancer
(SITC) toxicity management working group. J. ImmunoTher. Cancer 5:95. doi: 10.1186/s40425-017-0300-Z

Rodeghiero, F., Stasi, R., Gernsheimer, T., Michel, M., Provan, D., Arnold, D. M., et al. (2009). Standardization of terminology, definitions and outcome criteria in immune thrombocytopenic purpura of adults and children: report from an international working group. Blood 113, 2386-2396. doi: 10.1182/blood-200807- 162503

Sadaat, M., and Jang, S. (2018). Hemophagocytic lymphohistiocytosis with immunotherapy: brief review and case report. J. Immunother. Cancer 6:49. doi: 10.1186/s40425-018-0365-3

Schindler, K., Harmankaya, K., Kuk, D., Mangana, J., Michielin, O., Hoeller, C., et al. (2014). Correlation of absolute and relative eosinophil counts with immune-related adverse events in melanoma patients treated with ipilimumab. J. Clin. Oncol. 32, 9096. doi: 10.1200/jco.2014.32.15_suppl.9096

Schwab, K. S., Heine, A., Weimann, T., Kristiansen, G., and Brossart, P. (2016). Development of hemolytic anemia in a nivolumab-treated patient with refractory metastatic squamous cell skin cancer and chronic lymphatic leukemia. Case Rep. Oncol. 9, 373-378. doi: 10.1159/000447508

Shiuan, E., Beckermann, K. E., Ozgun, A., Kelly, C., Mckean, M., Mcquade, J., et al. (2017). Thrombocytopenia in patients with melanoma receiving immune checkpoint inhibitor therapy. J. Immunother. Cancer 5:8. doi: 10.1186/s40425017-0210-0

Simeone, E., Grimaldi, A. M., Esposito, A., Curvietto, M., Palla, M., Paone, M., et al. (2014). Serious haematological toxicity during and after ipilimumab treatment: a case series. J. Med. Case Rep. 8:240. doi: 10.1186/1752-1947-8-240

Solomon, L. R. (2015). Thrombocytopenia due to low-dose colchicine therapy: a possible drug interaction with nivolumab and implications for supportive care. Acta Oncol. 54, 1235-1237. doi: 10.3109/0284186x.2014.1002572

Sun, Y., Lee, S. K., Oo, T. H., and Rojas-Hernandez, C. M. (2018). Management of immune-mediated cytopenias in the era of cancer immunotherapy: a report of 4 cases. J. Immunother. 41, 32-34. doi: 10.1097/CJI.00000000000 00194

Tardy, M. P., Gastaud, L., Boscagli, A., Peyrade, F., Gallamini, A., and Thyss, A. (2017). Autoimmune hemolytic anemia after nivolumab treatment in hodgkin lymphoma responsive to immunosuppressive treatment. A. case report. Hematol. Oncol. 35, 875-877. doi: 10.1002/hon.2338

Wei, G., Nwakuche, U., Cadavid, G., Ajaz, A., Seiter, K., and Liu, D. (2012). Large granular lymphocytosis with severe neutropenia following ipilimumab therapy for metastatic melanoma. Exp. Hematol. Oncol. 1:3. doi: 10.1186/21623619-1-3

Wozniak, S., Mackiewicz-Wysocka, M., Krokowicz, L., Kwinta, L., and Mackiewicz, J. (2015). Febrile neutropenia in a metastatic melanoma patient treated with ipilimumab - case report. Oncol. Res. Treat. 38, 105-108. doi: 10.1159/ 000377650

Yuki, A., Takenouchi, T., Takatsuka, S., and Ishiguro, T. (2017). A case of pure red cell aplasia during nivolumab therapy for cardiac metastatic melanoma. Melanoma Res. 27, 635-637. doi: 10.1097/CMR.0000000000000392

Conflict of Interest Statement: RC is an employee of AstraZeneca Pharmaceuticals.

Copyright (c) 2019 Calvo. This is an open-access article distributed under the terms of the Creative Commons Attribution License (CC BY). The use, distribution or reproduction in other forums is permitted, provided the original author(s) and the copyright owner(s) are credited and that the original publication in this journal is cited, in accordance with accepted academic practice. No use, distribution or reproduction is permitted which does not comply with these terms. 